Research Article

\title{
Perilipin-I Level as Risk Marker of Insulin Resistance in Morbidly Obese Patients
}

\author{
Hanaa Addai Ali ${ }^{1}$, Ali Abbas Abo Algon ${ }^{2}$, Shukryia Shadhan Chyad ${ }^{3}$, Emad Abbas Jaffar Al-Mulla \\ ${ }^{1}$ Department of Chemistry, Faculty of Science, University of Kufa, P.O. Box 21, An-Najaf 54001, Iraq. \\ ${ }^{2}$ Pathological Department, Alkafeel University College, An-Najaf 54001, Iraq. \\ ${ }^{3}$ College of Health and Medical Techniques, Al-Furat Al-Awsat Technical University, 54003 Al-Kufa, Iraq. \\ Corresponding author. E-mail: almullaemad@gmail.com
}

Received: Sep. 16, 2017; Accepted: Nov. 6, 2017; Published: Nov. 30, 2017.

Citation: Hanaa addai Ali, Ali Abbas Abo Algon, Shukryia Shadhan Chyad, and Emad Abbas Jaffar Al-Mulla, Perilipin-1 Level as Risk Marker of Insulin Resistance in Morbidly Obese Patients. Nano Biomed. Eng., 2017, 9(4): 285-290.

DOI: 10.5101/nbe.v9i4.p285-290.

\begin{abstract}
Morbid obesity is a serious health condition that can interfere with basic physical functions such as breathing or walking. Those who are morbidly obese are at greater risk of illnesses including diabetes, high blood pressure, sleep apnea, gastro esophageal reflux disease, gallstones, osteoarthritis, heart disease, and cancer. This study was designed by taking 60 patients and 20 controls aged from 25 to 45 years with morbid obesity. A collection of samples was done by taking venous fasting blood samples from the patients and healthy volunteers after an overnight fasting. Insulin resistance (IR) was assessed using the homeostasis model assessment for insulin resistance (HOMA-IR), fasting blood glucose (FBG), glycated hemoglobin A1c ( $\mathrm{HbA} 1 \mathrm{c})$ and lipid profile kits were used to determine these parameters. It was observed that the increase level of perilipin-1 led to insulin resistance and hyperinsulinemia for 60 patients, while the level of perilipin-1 in 20 controls caused insulin sensitivity. The increase of all studied parameters was concluded from the p-value, which was less than 0.05 . The results also indicated that the level of perilipin- 1 could be considered a risk factor for many diseases. It could cause accumulation of the bad cholesterol in vascular tissues leading to atherosclerosis; it could cause changes in many factors in secretion, could cause insulin resistance and then diabetes mellitus. The level of fatty acid coming from continuous lipolysis causes fatty liver and live diseases.
\end{abstract}

Keywords: Obesity; Perilipin-1; Cholesterol; Body mass index (BMI); Lipid profile

\section{Introduction}

Obesity is generally defined as an increase in body weight from the normal rate due to accumulation of fat. This is due to eating a diet that contains higher calories than the body's ability to burn these calories to generate the energy needed for the body's activities. It is a chronic disease in which body fat has been accumulated in excessive level [1], which leads to adverse effects. Serious complications caused by morbidity and mortality, and the development of chronic diseases include atherosclerosis, liver fat, gallstones, diabetes, cancer, etc.

The obese person can be determined according to body mass index (BMI) factor. The persons with BMI $30-34$ can be classified as obesity type 1 , while those with BMI 35-39 are determined as type 2, and those with BMI higher than 40 are type 3 [3]. 
Perilipin is a protein that in humans coats lipid droplets in adipocytes, the fat-storing cells in adipose tissue [4]. Perilipin acts as a protective coating from the body's natural lipases, such as hormone-sensitive lipase, which breaks triglyceride (TG) into glycerol and free fatty acids for use in metabolism, a process called lipolysis [5].

The perilipin family consists of five proteins, Plin1, Plin2, Plin3, Plin4 and Plin5 [6]. Phosphorylation of perilipin is essential for the mobilization of fats in adipose tissue [7], and the major substrate for cAMP dependent protein kinase (protein kinase A (PKA)) in lipolytically stimulated adipocytes) [8]. Perilipin serves important functions in the regulation of basal and hormonally stimulated lipolysis [9]. Under basal conditions, perilipin restricts the access of cytosolic lipases to lipid droplets and thus promotes triacylglycerol storage [10]. In times of energy deficit, perilipin is phosphorylated by PKA and facilitates maximal lipolysis by hormone sensitive lipase and adipose TG lipase. Perilipin is hyperphosphorylated by PKA following $\beta$-adrenergic receptor activation. Phosphorylated perilipin changes conformation, exposing the stored lipids to hormone-sensitive lipase-mediated lipolysis [11]. Although PKA also phosphorylates hormone-sensitive lipase, which can increase its activity, the over 50-fold increase in fat mobilization (triggered by epinephrine) is primarily due to perilipin phosphorylation [5].

Complete hydrolysis of triacylglycerol involves the breakage of three ester bonds to liberate three fatty acids and a glycerol moiety [12]. The same enzyme, hormone sensitive lipase, is responsible for facilitating hydrolysis of the esters at positions 1 and 3 of the triacylglycerol. A second enzyme, 2-monoacylglycerol lipase, catalyzes hydrolysis of the remaining ester to yield a third free fatty acid and glycerol [13].

The aim of this study was to evaluate perilipin-1 with insulin resistance in morbid obese patients.

\section{Experimental}

\section{Study population}

A-Patients: Sixty patients participated in the study. Their age range was between 25 and 45 years with morbid obesity. The samples were collected from AlSadr Teaching Medical City in Najaf Governorate, Iraq and Royal Center for Jam during the period from December 2016 to May 2017.
A questionnaire was designed to obtain the information of the patient group, which included name, age, gender, height, weight, waist, hips, complication and other diseases such as hypertension, diabetes mellitus, other endocrine disorders and cardiovascular disease.

B-Controls: Twenty apparently healthy people were selected as the control group. Their age range was between 25 and 45 years, which was comparable to that of patients.

\section{Blood sample collection}

Venous fasting blood samples $(10 \mathrm{~mL})$ were collected from the patients and healthy volunteers after an overnight fasting. $2 \mathrm{~mL}$ blood samples were transferred to a tube containing ethylenediaminetetraacetic acid (EDTA), which was used for estimating glycated hemoglobin A1c (HbA1c) levels. The remaining $3 \mathrm{~mL}$ of blood was put at room temperature for 20 min for coagulation, after which the blood was put in centrifuge at $3000 \mathrm{rpm}$ for $10 \mathrm{~min}$. The serum was detached and brought into new plan tube and stored at $-20{ }^{\circ} \mathrm{C}$ prior to the biochemical tests to determine BMI $\left(\mathrm{kg} / \mathrm{m}^{2}\right)$, fasting blood glucose (FBG) (mg/dL), HbA1c (\%), perilipin-1 (ng/L) and lipid profile (mg/dL). Parameters such as fasting plasma glucose and lipid profile were measured through routine laboratory procedure. Blood glucose, cholesterol, high-density lipoprotein (HDL) cholesterol, TG and HbA1c were measured using photometrical method (Biolabo, France); perilipin-1 was measured by using the sandwich enzyme-linked immunosorbent assay (ELISA) (Elabsceince, China); insulin was analyzed by using ELISA (Demeditic, Germany).

\section{Statistical analysis}

In this research, SPSS V 23.0 was used to perform statistical analysis and to manage the database of this study. Normality was examined using KolmogorovSmirnov test. Descriptive information was depicted as mean \pm SD. Baseline and result factors were compared with paired $\mathrm{T}$ test. To assess the relationships of parameters following the operation, the Pearson correlation was utilized. A $p$ value of $<0.05$ was taken to express significant statistical difference.

\section{Results and Discussion}

The characteristics of the study groups are presented in Table 1, which consists of the data of both patients 
Table 1 Anthropometric and clinical parameters

\begin{tabular}{|c|c|c|c|c|c|c|c|}
\hline \multirow{2}{*}{ Parameter } & \multicolumn{3}{|c|}{ Patient $(n=60)$} & \multicolumn{4}{|c|}{ Control $(n=20)$} \\
\hline & Range & Mean & $\pm \mathrm{SD}$ & Range & Mean & $\pm \mathrm{SD}$ & P-value \\
\hline Weight (kg) & $130-160$ & 145 & \pm 14.21 & $60-71$ & 65 & \pm 6.12 & 0.001 \\
\hline Length $(\mathrm{cm})$ & $170-180$ & 175 & \pm 6.42 & $168-182$ & 177 & \pm 5.2 & 0.001 \\
\hline Waist (cm) & $110-130$ & 120 & \pm 8.30 & $83-88$ & 85 & \pm 2.2 & 0.001 \\
\hline Hips (cm) & $100-110$ & 105.2 & \pm 2.41 & $0.99-1.10$ & 92 & \pm 0.2 & 0.002 \\
\hline Waist/hips & $0.92-1.32$ & 1.12 & \pm 0.2 & $0.72-0.89$ & 0.85 & \pm 0.31 & 0.001 \\
\hline BMI $\left(\mathrm{kg} / \mathrm{m}^{2}\right)$ & $40-51$ & 45 & \pm 5.21 & 20.3-24.31 & 22.00 & \pm 2.21 & 0.001 \\
\hline TG (mg/dL) & $122-162$ & 140.72 & \pm 22.57 & 20.00 & 50.25 & \pm 5.42 & 0.001 \\
\hline HDL (mg/dL) & $22.5-31.2$ & 26.61 & \pm 4.37 & 26.60 & 113.04 & \pm 8.30 & 0.001 \\
\hline LDL (mg/dL) & $145.3-175.2$ & 159.20 & \pm 15.04 & 9.55 & 14.72 & \pm 2.41 & 0.002 \\
\hline VLDL (mg/dL) & 22.6-33.5 & 28.21 & \pm 5.11 & 30.00 & 175.98 & \pm 7.75 & 0.001 \\
\hline Cholesterol (mg/dL) & 202.3-233.6 & 220.33 & \pm 18.20 & 0.98 & 175.96 & \pm 0.21 & 0.003 \\
\hline HbA1c (mg/dL) & 7.68 & 8.37 & \pm 2.06 & 31.87 & 92.38 & \pm 10.21 & 0.002 \\
\hline FBG (mg/dL) & 121.00 & 147.86 & \pm 25.85 & 0.81 & 75.93 & \pm 0.24 & 0.001 \\
\hline IR (mg/dL) & 16.47 & 10.63 & \pm 4.40 & 1.09 & 2.75 & \pm 0.34 & 0.003 \\
\hline Perilipin-1 (ng/dL) & 5.79 & 4.19 & \pm 1.10 & 5.51 & 17.18 & \pm 1.13 & 0.001 \\
\hline
\end{tabular}

with morbid obesity and the control group. They include the number, BMI, lipid profile, FBG, HbA1c and perilipin-1.

Levels of biochemical parameters of the 60 enrolled patients were summarized in Table 1. Significant increases of the levels of fasting blood glucose ( $\mathrm{p}=$ $0.001)$, insulin resistance (IR) ( $\mathrm{p}=0.003)$, perilipin-1 $(p=0.001)$, cholesterol $(p=0.003)$, triglycerides $(p$ $=0.001)$, low-density lipoprotein $(\mathrm{LDL})(\mathrm{P}=0.002)$, very-low-density lipoprotein $(\mathrm{VLDL})(\mathrm{P}=0.001)$, HDL $(\mathrm{P}=0.001)$ and HbA1c $(\mathrm{p}=0.002)$ were obtained in morbid obesity patients' group when compared with those of the control group.

It was seen in Table 1 that the mean value of each BMI for patients ( $\left.45 \pm 5.21 \mathrm{~kg} / \mathrm{m}^{2}\right)$ and for controls (22 $\left.\pm 2.21 \mathrm{~kg} / \mathrm{m}^{2}\right)$ was significant $(\mathrm{p}=0.001)$ compared to controls and patients [12].

As illustrated in Fig. 1, the levels of perilipin-1 increased as the body mass increased [14]. This increase was due to that patients with high BMI (more than 40) had higher percent of lipid, and therefore they needed to depose it in their adipose tissues, for which reason high level of perilpin-1 was required to protect lipid droplet in adipose tissues (Fig. 1, Table 2) [15].

Fig. 2 \& 3 demonsterate the relationship between blood sugar and HbA1c with relation to perilipin-1. It could be concluded that the blood glucose level (Fig. 2, Table 3) and HbA1c (Fig. 3, Table 4) would be

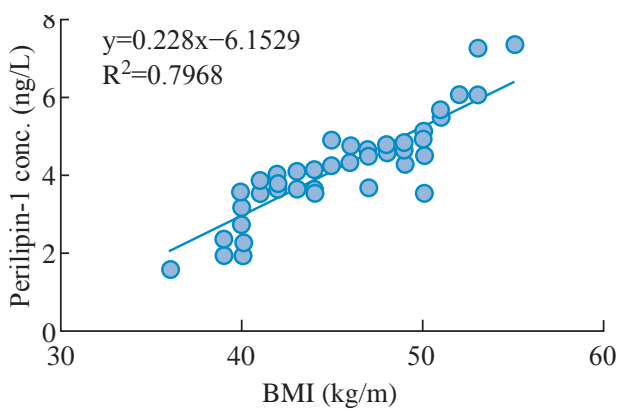

Fig. 1 Levels of perilipin-1 with relation to BMI.

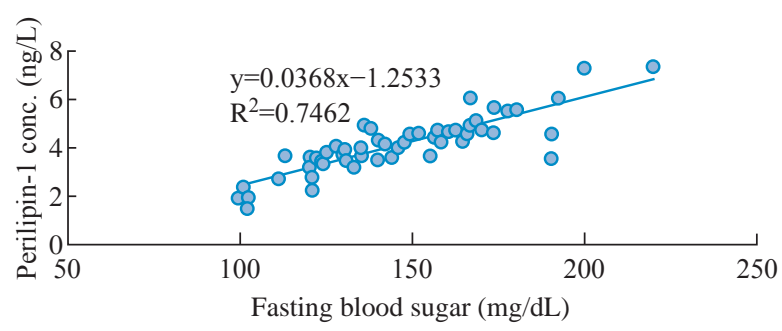

Fig. 2 The relationship between perilipin-1 and blood sugar.

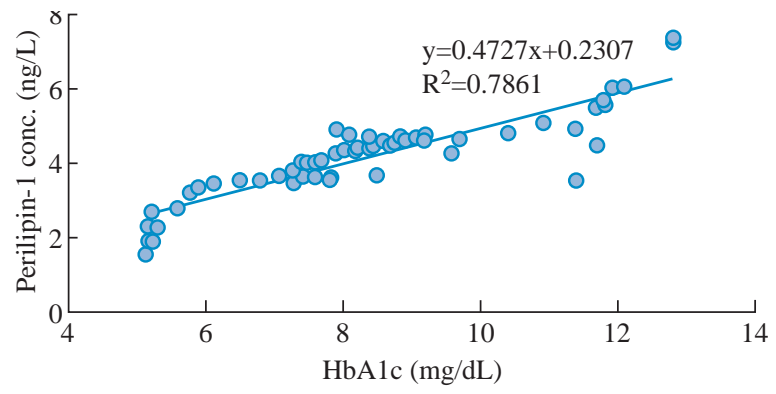

Fig. 3 The relationship between perilipin-1 and HbA1c. 


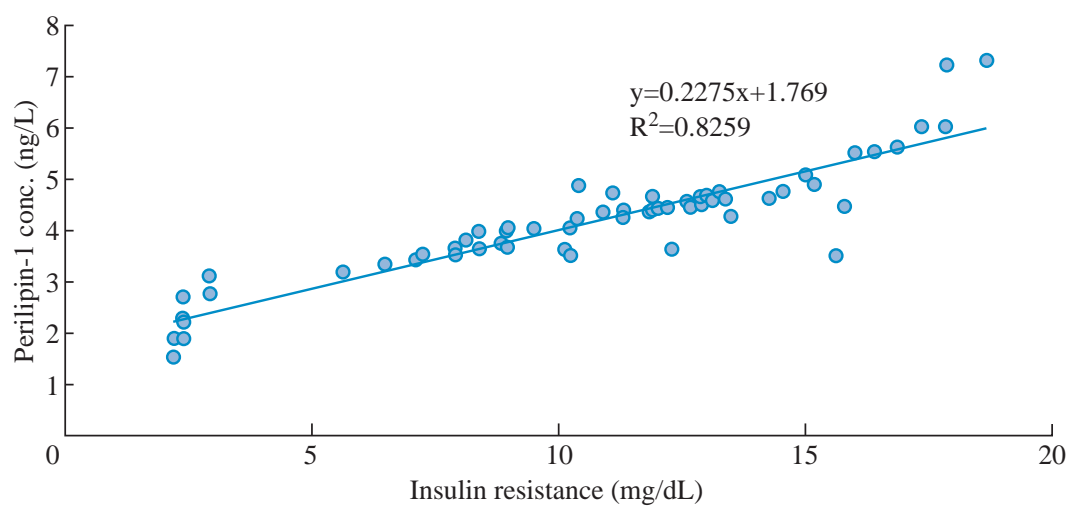

Fig. 4 The relationship of perilipin with insulin resistance.

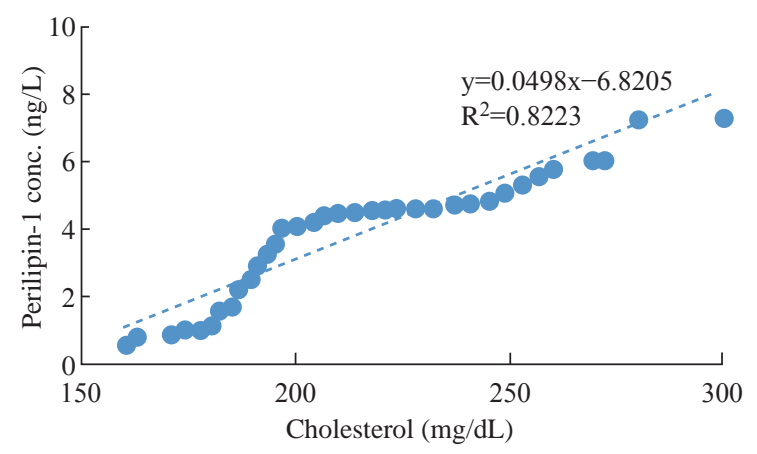

Fig. 5 The relationship between perilipin-1 and cholesterol.

Table 2 Results of linear regression analysis for perilipin-1 level with BMI of morbid obese patients

\begin{tabular}{ccc}
\hline & $\mathrm{r}$ & $\mathrm{p}$ \\
\hline Perilipin-1 & 0.89 & 0.001 \\
\hline
\end{tabular}

r: rank correlation coefficient

p: probability value

Table 3 Results of linear regression analysis for perilipin-1 level with relation to fasting blood sugar of morbid obese patients

\begin{tabular}{ccc}
\hline & $\mathrm{r}$ & $\mathrm{p}$ \\
\hline Perilipin-1 & 0.86 & 0.001 \\
\hline
\end{tabular}

$\mathrm{r}$ : rank correlation coefficient

p: probability value

Table 4 Results of linear regression analysis for perilipin-1 level with relation to HbA1c of morbid obese patients

\begin{tabular}{ccc}
\hline & $\mathrm{r}$ & $\mathrm{p}$ \\
\hline Perilipin-1 & 0.88 & 0.001 \\
\hline
\end{tabular}

r: rank correlation coefficient

p: probability value

Table 5 Results of linear regression analysis for perilipin-1 level with relation to insulin resistance of morbid obese patients

\begin{tabular}{ccc}
\hline & $\mathrm{r}$ & $\mathrm{p}$ \\
\hline Perilipin-1 & 0.90 & 0.001 \\
\hline
\end{tabular}

$\mathrm{r}$ : rank correlation coefficient

p: probability value

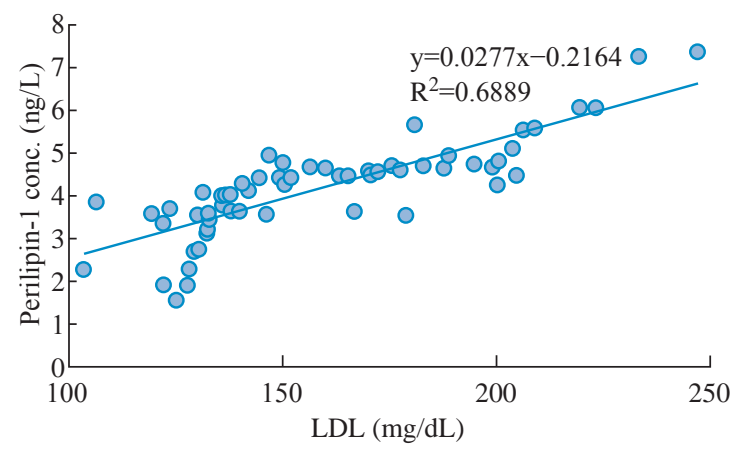

Fig. 6 The relationship between perilipin-1 and LDL. elevated in those paitents as a result of IR means which arised due to high level of perilipin-1 (Fig. 4, Table 5) [16] .

The lipid profile parameters and their relationships with perilipin levels were studied in the plasma [17]. As shown in Fig. 5, a high level of perilipin-1 would induce insulin resistance which would be an indicator for low energy level inside cells (Table 6), and this mark would activate hormomes of fasting such as glucagon [18]. In Fig. 6, the hormonal state would induce lypolysis inside adipose tissues which would export free fatty acid and glycerol to the plasm to be

Table 6 Results of linear regression analysis for perilipin-1 level with relation to cholesterol of morbid obese patients

\begin{tabular}{ccc}
\hline & $\mathrm{r}$ & $\mathrm{p}$ \\
\hline Perilipin-1 & 0.91 & 0.001 \\
\hline
\end{tabular}

r: rank correlation coefficient

p: probability value

Table 7 Results of linear regression analysis for perilipin-1 level with relation to LDL of morbid obese patients

\begin{tabular}{ccc}
\hline & $\mathrm{r}$ & $\mathrm{p}$ \\
\hline Perilipin-1 & 0.82 & 0.001 \\
\hline
\end{tabular}

r: rank correlation coefficient

p: probability value 


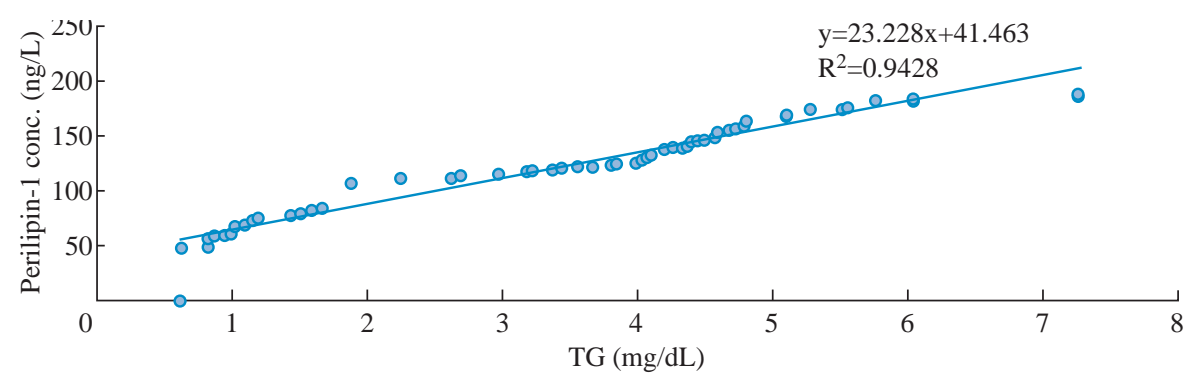

Fig. 7 The relationship between perilipin-1 and TG.

used as an alternative source for energy (Table 7) [12]. As blood glucose level was elevatied and remained circulating in the plasma, the liver would utilze it in the minor pathways of glucose due to high energy level coming from beta-oxidation [19]. These minor pathways include include denovo synthesis of fatty acid and cholesterol [4]. As dmonstrated in Fig. 7, the liver would export minor pathway products as a lipoprotein in the blood [5]. VLDL would be in high ratio in the blood, and during its circulation, an enzyme which was found in the walls of blood vesels would hydrolyze TG to free fatty acid and glycerol (Table 8), leaving cholesterol in high ratio inside lipoprotein ball which is called LDL [13]. This would make the ratio of LDL high in blood which consisted of cholestrol [20]. Fig. 8 and Table 9 reveal the result that the total cholestrol would rise with a high level of perilipin-1

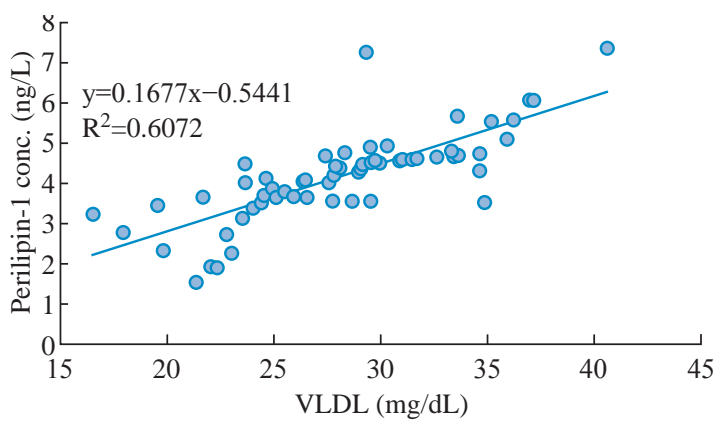

Fig. 8 The relationship between perilipin-1 and VLDL.

Table 8 Results of linear regression analysis for perilipin-1 level with relation to TG of morbid obese patients

\begin{tabular}{ccc}
\hline & $\mathrm{r}$ & $\mathrm{p}$ \\
\hline Perilipin-1 & 0.96 & 0.001 \\
\hline
\end{tabular}

r: rank correlation coefficient

p: probability value

Table 9 Results of linear regression analysis for perilipin-1 level with relation to VLDL of morbid obese patients

\begin{tabular}{ccc}
\hline & $\mathrm{r}$ & $\mathrm{p}$ \\
\hline Perilipin-1 & 0.77 & 0.001 \\
\hline
\end{tabular}

r: rank correlation coefficient

p: probability value
[21].

\section{Conclusions}

In this research, it was noticed that protein that covered lipid droplet (perilipin-1) was in direct proportion with the amount of stored fat of adipocytes in adipose tissues. Change of perilipin-1 level was positively correlated with BMI, FBG, HbA1c, IR and lipid profile. Our statistical data showed how the level of the perilipin-1 changed with BMI, FBG, HbA1c, IR and lipid profile with comparison between patients and the control group. In addition, the level of perilipin-1 could be considered as a risk factor for many diseases such as diabetes, mellitus, atherosclerosis and fatty liver.

\section{Conflict of Interests}

The authors declare that no competing interest exists.

\section{References}

[1] U.J. Jung, M.S. Choi, Obesity and its metabolic complications: the role of adipokines and the relationship between obesity, inflammation, insulin resistance, dyslipidemia and nonalcoholic fatty liver disease. Int. J. Mol. Sci., 2014, 15: 6184-6223.

[2] R.F. Aten, K.M. Duarte, and H.R. Behrman, Regulation of ovarian antioxidant vitamins, reduced glutathione, and lipid peroxidation by luteinizing hormone and prostaglandin F2 $\alpha$. Biol Reprod., 1992, 46: 401-407.

[3] R.R. Al-Araji, M.S. Mashkour, and E.A.J. Al-Mulla, Spectrophotometric determination of vitamin folic acid B9 in some drugs using 1, 2-naphthoquine-4-sulphonate (NQS). Nano Biomed. Eng., 2017, 9(3): 208-213.

[4] D.L. Brasaemle, Perilipin A increases triacylglycerol storage by decreasing the rate of triacylglycerol hydrolysis. J. Biol. Chem., 2000, 275: 38486-38493.

[5] J.R. Skinner, L.-A.L.S. Harris, T.M. Shew, et al., Perilipin 1 moves between the fat droplet and the endoplasmic reticulum. Adipocyte, 2013, 2: 80-86.

[6] H.K. Al-Hakeim, R.S. Al-Zabeba, and E. Grulke, Interaction of calcium phosphate nanoparticles with 
human chorionic gonadotropin modifies secondary and tertiary protein structure. Nova Biotechnologica et Chimica, 2015, 14 (2): 141-157.

[7] M.M. Radhi, Z.N. Hamed, and S.S. Ezzaldeen, Effect of micro- and nanoparticles of ampicillin trihydrate on blood medium: A voltammetric study. Nano Biomed. Eng., 2017, 9(3): 185-190.

[8] M.H. Moon, Antiobesity activity of a sphingosine 1-phosphate analogue FTY720 observed in adipocytes and obese mouse model. Experimental \& Molecular Medicine, 2012, 44: 603.

[9] Y.K. Amir, M.M. Radhi, Use of nano-sensors of the interferences between $\mathrm{Pb}(\mathrm{II})$ with each of $\mathrm{Mg}(\mathrm{II}), \mathrm{Zn}(\mathrm{II})$, $\mathrm{Mn}(\mathrm{II}), \mathrm{Ca}(\mathrm{II}), \mathrm{Co}(\mathrm{II})$ and PO4-3 in blood medium: An Electrochemical study. Nano Biomed. Eng., 2017, 9(3): 199-207.

[10] K.H. Gathwan, I.T. Al-Karkhi, Hepatic toxicity of nickel chloride in mice. Res. Chem. Intermed., 2013, 39(6): 2537-2542.

[11] R.E. Macpherson, Skeletal muscle PLIN3 and PLIN5 are serine phosphorylated at rest and following lipolysis during adrenergic or contractile stimulation. Physiol Rep 1, 2013, 84: 34-41.

[12] T. Sawada, Perilipin overexpression in white adipose tissue induces a brown fat-like phenotype. PLoS One, 2010, 5: 22-29.

[13] S.K. Malin, J. Bena, B. Abood, et al. Attenuated improvements in adiponectin and fat loss characterize type 2 diabetes non-remission status after bariatric surgery. Diabetes, Obes. Metab., 2014, 16: 1230-1238.

[14] J. Buteau, S. Foisy, C.J. Rhodes, et al., Protein kinase Czeta activation mediates glucagon-like peptide-1induced pancreatic beta-cell proliferation. Diabetes, 2001,
50: 2237-2243.

[15] J. Bi, Y. Xiang, H. Chen, et al., Opposite and redundant roles of the two Drosophila perilipins in lipid mobilization. Journal of Cell Science, 2012, 125: 35683577.

[16] A. Afatoonian, H. Oskouian, S. Ahmadi, et al., Adipocytokine orosomucoid integrates inflammatory (2-6 mm). J Assist Reprod Genet, 2009, 26: 319-325.

[17] K. Alberti, Definition, diagnosis, and classification of diabetes mellitus and its complications. Part I: Diagnosis and classification of diabetes mellitus provisional report of a WHO consultation. Diabetic Med., 1998, 15: 539553.

[18] J. Buyse, Adipose tissue and lipid metabolism. Sturkie's Avian PhysioL., 2015, 23: 443-453.

[19] B.J. Ashkenazi, Maternal obesity induces epigenetic modifications in overweight women. Reprod Biomed Online, 2010, 21: 706-711.

[20] J.T. Tansey, C. Sztalryd, E.M. Hlavin, et al., The central role of perilipin a in lipid metabolism and adipocyte lipolysis. IUBMB life, 2004, 56: 379-385.

[21] S.M. Adams, V. Terry, M.J. Hosie, et al., The perilipin family of structural lipid droplet proteins regulated and natural cycles. Reprod Biol Endocrinol., 2004, 2: 21-29.

Copyright $\subseteq$ Hanaa addai Ali, Ali Abbas Abo Algon, Shukryia Shadhan Chyad, and Emad Abbas Jaffar Al-Mulla, This is an open-access article distributed under the terms of the Creative Commons Attribution License, which permits unrestricted use, distribution, and reproduction in any medium, provided the original author and source are credited. 